\title{
Aphasie bei Kindern
}

\author{
Patienteninformation
}

Aphasie bei Kindern ist eine erworbene Sprachbehinderung, die infolge einer Schädigung des Gehirns durch Schädelhirntrauma, Schlaganfall, Tumor oder entzündliche Erkrankungen (z.B. Hirnhautentzündungen) in unterschiedlicher Ausprägung auftreten kann. Das SchädelHirn-Trauma nach Unfällen gilt mit 80\% als Hauptursache für Aphasie im Kindesalter. Kinder erleiden Schädel-Hirn-Traumata am häufigsten durch Unfälle im Straßenverkehr, Stürze bei Spiel und Sport oder auch als Folge körperlicher Gewalt.

Auch Kinder, die die Sprachentwicklung noch nicht abgeschlossen haben, können eine Aphasie erleiden. Allerdings muss in diesem Zusammenhang betont werden, dass es sich bei einer Aphasie um einen Abbau oder Verlust vorhandener Sprachfunktionen handelt. Darin zeigt sich ein wesentlicher Unterschied zu Sprachentwicklungsstörungen. Deshalb spricht man bei Kindern erst ab einem Alter von etwa 1,5 bis 2 Jahren von Aphasie, wenn die Sprachentwicklung auf Mehrwortebene bereits begonnen hat.

\section{Symptome und begleitete Erkrankungen \\ $\nabla$}

Wie bei Jugendlichen und Erwachsenen wirkt sich die Aphasie bei Kindern auf die unterschiedlichen sprachlichen Ebenen (Verstehen, Sprechen, Lesen, Schreiben) aus. Kinder mit Aphasie zeigen direkt nach der Hirnschädigung häufig eine Zeit des völligen Schweigens. Im Anschluss entwickeln die meisten Kinder mit Aphasie eine reduzierte Spontansprache, jedoch äußert sich jede Aphasie anders. Mittlerweile wurde nachgewiesen, dass bei Kindern ähnlich vielfältige Symptome auftreten können wie bei Erwachsenen. In Bezug auf die Genesung und die sprachlichen Fähigkeiten von Aphasien bei Kindern gilt, dass Prognose und Verlauf im Einzelfall sehr variieren. Einheitliche Aussagen sind daher schwierig.
Aphasien im Kindesalter treten selten isoliert auf. Aufgrund der erworbenen Hirnschädigung sind Begleitsymptome wie Halbseitenlähmungen häufig. Auch Sprechstörungen (Dysarthrie, Sprechapraxie), Epilepsien und Dysphagien (Schluckstörungen) können auftreten. Ist das Frontalhirn mitbetroffen, kommt es nicht selten zu Konzentrations-, Aufmerksamkeits- und Gedächtnisstörungen. Verhaltensauffälligkeiten, Aggressionen und in manchen Fällen auch Hyperaktivität werden besonders im schulischen Kontext beobachtet. In Studien konnte jedoch gezeigt werden, dass diese Verhaltensauffälligkeiten in vielen Fällen zurückgehen, wenn den Kindern alternative Kommunikationssysteme angeboten werden.

\section{Diagnostik und Therapie \\ $\nabla$}

Ziel der Diagnostik ist es, ein möglichst umfassendes Bild der sprachlichen Fähigkeiten des Kindes zu bekommen, um daran therapeutisch anknüpfen zu können. Dazu gehört u. a. die Erfassung des sprachlichen Niveaus vor der Hirnschädigung.

Wesentliche Therapieaufgaben sind die Wiederherstellung jener sprachlichen Fähigkeiten, über die das Kind vor der Schädigung verfügte. Kinder mit Aphasie benötigen viel Unterstützung beim Erlernen neuer Fähigkeiten in der Schule. Lesen und Schreiben wird in der Regel nur schwer erworben.

\section{Tipps für betroffene Familien $\nabla$}

Aphasie mit ihren vielfältigen Begleitsymptomen hat immer eine Veränderung des gesamten familiären Lebens zur Folge. Lassen Sie sich nicht entmutigen, wenn immer wieder Fähigkeiten verloren gehen, die bereits wiedererworben wurden. Je nach Tagesform schwanken die Fähigkeiten bei aphasischen Kindern. Ebenfalls zeigt sich, dass bei entsprechender Förderung auch noch in der sog. chronischen Phase Verbesserungen möglich sind.

Hilfreich ist, wenn Sie sich und anderen klar machen, dass eine Sprachbehinderung wie Aphasie keine Denkstörung oder geistige Behinderung ist! Auch wenn sich Ihr Kind nicht altersgemäß sprachlich ausdrücken kann, so heißt das nicht, dass es nicht versteht, was Sie ihm sagen - vorausgesetzt es liegt keine Sprachverständnisstörung vor. Intelligenz und Denken sind bei einer Aphasie nicht betroffen!

Achten Sie bei der Beschulung, aber auch im Alltag, darauf dass sowohl Über- als auch Unterforderung bei Ihrem Kind zu Verhaltensauffälligkeiten führen kann. Lassen Sie sich durch das ungewohnte Verhalten Ihres Kindes nicht entmutigen und suchen Sie gemeinsam mit Ihrem Kind und der Unterstützung von Fachleuten in speziellen neurologischen Rehakliniken nach dem richtigen Förderlevel.

\section{Fazit}

Aphasie bei Kindern ist eine erworbene Sprachbehinderung, die sich auf die unterschiedlichen sprachlichen Ebenen auswirkt und die mit ihren vielfältigen Begleitsymptomen immer eine Veränderung des gesamten familiären Lebens zur Folge hat. Sie ist aber keine Denkstörung oder geistige Behinderung und bei entsprechender Förderung sind auch noch in der chronischen Phase Verbesserungen möglich.

\section{Melanie Kubandt, Würzburg}

überreicht durch Thieme und 